\title{
Dynamic Contrast-Enhanced Perfusion Processing for Neuroradiologists: Model-Dependent Analysis May Not Be Necessary for Determining Recurrent High-Grade Glioma versus Treatment Effect
}

\author{
J.D. Hamilton, J. Lin, C. Ison, N.E. Leeds, E.F. Jackson, G.N. Fuller, L. Ketonen, and A.J. Kumar
}

\begin{abstract}
BACKGROUND AND PURPOSE: Dynamic contrast-enhanced perfusion MR imaging has proved useful in determining whether a contrastenhancing lesion is secondary to recurrent glial tumor or is treatment-related. In this article, we explore the best method for dynamic contrast-enhanced data analysis.
\end{abstract}

\begin{abstract}
MATERIALS AND METHODS: We retrospectively reviewed 24 patients who met the following conditions: 1) had at least an initial treatment of a glioma, 2) underwent a half-dose contrast agent (0.05-mmol/kg) diagnostic-quality dynamic contrast-enhanced perfusion study for an enhancing lesion, and 3) had a diagnosis by pathology within 30 days of imaging. The dynamic contrast-enhanced data were processed by using model-dependent analysis (nordicICE) using a 2-compartment model and model-independent signal intensity with time. Multiple methods of determining the vascular input function and numerous perfusion parameters were tested in comparison with a pathologic diagnosis.
\end{abstract}

RESULTS: The best accuracy (88\%) with good correlation compared with pathology $(P=.005)$ was obtained by using a novel, modelindependent signal-intensity measurement derived from a brief integration beginning after the initial washout and by using the vascular input function from the superior sagittal sinus for normalization. Modeled parameters, such as mean endothelial transfer constant $>0.05$ minutes $^{-1}$, correlated $(P=.002)$ but did not reach a diagnostic accuracy equivalent to the model-independent parameter.

CONCLUSIONS: A novel model-independent dynamic contrast-enhanced analysis method showed diagnostic equivalency to more complex model-dependent methods. Having a brief integration after the first pass of contrast may diminish the effects of partial volume macroscopic vessels and slow progressive enhancement characteristic of necrosis. The simple modeling is technique- and observerdependent but is less time-consuming.

ABBREVIATIONS: $\mathrm{AUC}=$ area under the curve; $\mathrm{DCE}=$ dynamic contrast-enhanced perfusion $\mathrm{MRI} ; K^{\mathrm{trans}}=$ endothelial transfer constant; $\mathrm{SSS}=$ superior sagittal sinus; $\mathrm{VIF}=$ vascular input function; $v_{\mathrm{p}}=$ vascular plasma volume fraction

$\mathbf{T}$ here are over 23,000 newly diagnosed malignant brain tumors in the United States every year. ${ }^{1}$ Glioblastoma is one of the most common and devastating brain tumors. Most patients survive $<1$ year from diagnosis if untreated. Despite the large num-

Received September 13, 2012; accepted after revision August 27, 2014.

From the Department of Diagnostic Radiology, Section of Neuroimaging (J.D.H., C.I., N.E.L., L.K., A.J.K.), Department of Imaging Physics, Section of MRI Physics (J.L., E.F.J.), and Department of Pathology, Section of Neuropathology (G.N.F.), The University of Texas M.D. Anderson Cancer Center, Houston, Texas; Radiology Partners Houston (J.D.H.), Houston, Texas; Rice University (J.L.), Houston, Texas; and Baylor College of Medicine (J.L.), Houston, Texas.

This work was supported by the M.D. Anderson Levit Distinguished Chairman Funds.

Paper previously presented at: Annual Meeting of the American Society Neuroradiology, June 4-9, 2011; Seattle, Washington.

Please address correspondence to Jackson D. Hamilton, MD, Radiology Partners Houston, 5301 Hollister Dr, Suite 350, Houston, TX 77040; e-mail:

Jackson.hamilton@radpartners.com

http://dx.doi.org/10.3174/ajnr.A4190 ber of potential treatments that have been used, only modest gains in mortality (9-12 months of life gained) have been made due to tumor recurrence. ${ }^{2}$ A variety of treatment-related changes occur on normal brain tissues related to the effects of radiation and chemotherapy, such as temozolomide. ${ }^{3}$ Delayed radiation-/treatment-induced brain necrosis is one of the effects that can cause an enlarging, enhancing mass. This can mimic recurrent tumor on conventional CT or MR imaging, with few clues as to the diagnosis. ${ }^{4}$

Delayed radiation necrosis may be a result of progressive, obliterative endarteritis resulting in hypoxia, ${ }^{5}$ with an immune inflammatory response. Treatments include steroids, hyperbaric oxygen, ${ }^{5}$ bevacizumab (antivascular endothelial growth factor) in resistant cases, ${ }^{6,7}$ or surgical resection for pending herniation. With recurrent gliomas (astrocytomas and oligodendrogliomas, most commonly glioblastoma), single agent or combination chemotherapy and/or additional surgery or radiation therapy may be sought. ${ }^{8}$ 
MR perfusion techniques, such as dynamic contrast-enhanced (DCE) and dynamic susceptibility contrast perfusion examinations, have been shown to be useful in differentiating delayed radiation-induced necrosis from recurrent glial tumor. ${ }^{9-12} \mathrm{~A}$ combined parameter approach further improves accuracy. ${ }^{13}$ DCE data can be either model-independent or model-dependent. Model-independent indices rely on the T1 signal intensity increase by using gadolinium contrast. In the initial first pass of contrast (vascular phase), the signal change is related to the blood volume in the tissue of interest. ${ }^{9}$ After the concentration of gadolinium in the blood begins to equilibrate with the second and third passes of contrast, the leakage of contrast from the vessel to the interstitial space (extravascular, extracellular space) becomes an increasingly important factor in the signal-intensity enhancement. Various model-independent parameters by using the area under the curve of signal intensity with time have been proposed in the pharmacologic literature. ${ }^{14}$

Model-dependent indices are often derived from a 2-compartment model. ${ }^{15}$ In this model or its derivatives, the intravascular and interstitial spaces are treated as having a certain volume fraction (eg, $v_{\mathrm{p}}$ [vascular plasma volume fraction]) with both forward (endothelial transfer constant $\left[K^{\text {trans }}\right]$ ) and backward constants to explain the kinetics across a membrane (ie, the blood-brain barrier). These values are derived from a deconvolution that compares the dynamic T1 signal changes with contrast in a region of interest with a vascular input function (VIF). ${ }^{16}$ There is considerable variability as to the best method to choose the VIF, either by using an artery or a vein or having an algorithm that chooses the pixels by curve-fitting clusters of pixels. ${ }^{17}$ There has been recent debate on whether simple model-independent analysis ${ }^{10}$ of signal intensity with time may perform as well as more complex compartmental models. ${ }^{9,18}$ The following article seeks the best DCE analysis for improving the accuracy of determining delayed radiation-induced necrosis versus recurrent glioma, by using various model-dependent and -independent parameters and VIF methodology.

\section{MATERIALS AND METHODS Patient Population}

With institutional review board approval, the data from patients were retrospectively gathered from January 2009 to January 2012. Inclusion criteria required that the patient have the following: 1) prior diagnosis of glioma that had been previously treated, including conventional standards of radiation therapy and usually temozolomide; 2) parenchymal brain enhancement that progressed over successive imaging studies; 3) diagnostic quality protocol including a half-dose contrast agent DCE protocol $(0.05 \mathrm{mmol} / \mathrm{kg}$ at rate $4 \mathrm{~mL} / \mathrm{s}$ ); 4) biopsy or resection within 30 days of the protocol; and 5) older than 18 years of age. Demographic and treatment data were recorded.

\section{Imaging Acquisition}

All imaging data were obtained on an HDxt 3T MR imaging scanners by using a multichannel phased array head coil (GE Healthcare, Milwaukee, Wisconsin). Following any required standardof-care precontrast agent imaging, DCE data were acquired by using a T1-weighted 3D fast-spoiled gradient-echo technique.
Images were acquired with a temporal resolution of 5.1 seconds before, during, and up to 4 minutes after administration of $0.05-\mathrm{mmol} / \mathrm{kg}$ gadopentetate dimeglumine (Magnevist; Bayer HealthCare Pharmaceuticals, Wayne, New Jersey) followed by a 20-mL saline flush. These were injected by an MR imaging-compatible automated system at $4 \mathrm{~mL} / \mathrm{s}$. Half-dose DCE was performed before subsequent full-dose dynamic susceptibility contrast perfusion imaging as part of the imaging protocol. DCE parameters included the following: TE, $1.8 \mathrm{~ms}$; TR, $4 \mathrm{~ms} ; 256 \times$ 160 matrix; $24 \times 18 \mathrm{~cm}$ FOV; sixteen 5 -mm thick contiguous sections ( 8 -cm coverage); and total scan time of approximately 5 minutes. This imaging yielded approximately 36 time points with a reconstructed voxel resolution of approximately $1.0 \times 1.0 \times 5.0$ $\mathrm{mm}$. The MR imaging examination concluded with any necessary standard-of-care postcontrast agent-enhanced imaging.

\section{Image Processing}

DCE Analysis Method 1. A 2-compartment generalized kinetic model ${ }^{15}$ was used with the technique proposed by Murase et al, ${ }^{16}$ including motion correction as implemented in the software package nordicICE (NordicNeuroLab, Bergen, Norway). A 3-parameter analysis was performed to yield values of endothelial transfer constant related to contrast leaving the vessel, the reflux rate constant related to contrast re-entering the vessel, and the vascular plasma volume fraction related to the percentage volume of blood vessels. The values of the extravascular, extracellular volume fraction (interstitial space) were then computed from the ratio of $K^{\text {trans }}$ over the reflux rate constant. Recorded values included mean and maximum. In addition, model-independent parameters (ie, area under the curve [AUC], initial AUC, and maximum slope) were also computed with both scaled and unscaled values to the VIF.

Several methods for detecting the VIF were tested. With a previously described algorithm, ${ }^{17}$ which picked 5 clusters of voxels that are expected to be vessels on the basis of the dynamic signal response, VIFs were generated from the following: 1) whole-brain images; 2) a region of interest confined to the vertical portion of the superior sagittal sinus (SSS), which avoided feeding veins causing mixing artifacts in the same section (further described in method 2 below); and 3) a region of interest surrounding the M1 to M2 bifurcation of the MCA ipsilateral to the lesion. Additionally, a fourth method was used with all pixels in a region of interest placed in the SSS. A deconvolution algorithm was used to generate maps of the parameters of interest. ${ }^{16}$ Oval lesion ROIs were placed over the area of enhancement by a radiologist (J.D.H.) and trainee (J.L.), blinded to the pathology results. The lesion regionof-interest locations were maintained through the multiple processing options with different VIFs. The values were recorded for the axial image with the maximum $K^{\text {trans }}$ value.

DCE Analysis Method 2. A simple, model-independent analysis evaluated the relative change in signal intensity with time. The AUC was an integration of the enhancing region of interest of the lesion divided by the VIF (Advantage Workstation, Version 4.3 or 4.4; GE Healthcare). The VIF from a 1-cm-diameter oval region of interest was placed in the SSS. This region of interest was positioned in the vertical portion of the SSS, while avoiding feeding 
veins entering the sinus at the same level, to decrease inhomogeneity from mixing artifacts. The lesion ROIs were defined by the contrast-enhancing area with the most signal change and contoured by 2 board-certified radiologists with neuroradiology fellowship training (J.D.H. and C.I.), blinded to the pathology results.

Multiple limits of the average AUC integration were evaluated. The starting time for VIF integration was the start of the first-pass contrast agent wash-in or at the end of contrast agent washout. The end time of integration was computed at 30,60, 90, or 120 seconds after the arrival of the contrast bolus (wash-in). Additionally, an integration of just the time of the first pass of contrast agent (wash-in to washout) was used (similar timing to that of dynamic susceptibility plasma volume measurements). The region-of-interest positions were maintained across all time iterations for the AUC. Care was taken to avoid large central nonenhancing areas (due to cyst or necrosis) and medium-to-large vessels (by using source images from contrast-enhanced, T2, and gradient-echo images). The average values of the AUC for both the VIF and the user-defined area of contrast enhancement were recorded. The contrast-enhanced lesion AUC value was divided by the VIF AUC value and expressed as a percentage for each patient.

\section{Pathologic Data}

After imaging, the selected patients underwent either stereotactic biopsy or resection within 30 days of imaging as part of their clinical care. Medical records and follow-up imaging data were also followed to minimize the possibility of false pathologic results due to tissue undersampling. Pathology results were graded on a 5-point scale based on the pathology report rendered by a boardcertified pathologist specializing in brain tumors. This ranged from nearly completely tumor (score $=1$ ) to complete necrosis or other treatment-related effects $($ score $=5$ ). Scores of 3 (small areas [ $\sim 5$ cells per high-power field] of viable tumor) and 4 (predominate necrosis with scattered atypical cells or rare mitosis, which did not progress within 6 months) were important because they delineated the categorization of recurrent tumor versus necrosis, respectively. Each pathology report was independently scored by 2 evaluators (J.D.H. and G.N.F.). In case of disagreement or an equivocal report, the pathology sample was re-evaluated by a board-certified neuropathologist (G.N.F.).

\section{Statistics}

The results of these 2 methods of perfusion analysis from the same datasets were compared with the pathology results. The Spearman nonparametric 2-tailed correlation was used to compare the perfusion data with the categoric ranked pathology grading, by using SPSS (IBM, Armonk, New York) and R, Version 3.0.1 (http:// www.r-project.org). Additionally, post hoc arbitrary values were used to find optimal cutoff values for sensitivity, specificity, and accuracy, by using the cutoff in pathology grading of 3 and 4, as defined above. The Kaplan-Meier method was used to estimate the distribution of overall survival times from the time of the MR imaging examination, and groups were compared by using the log-rank test. Interrater reliability was calculated as both a per-

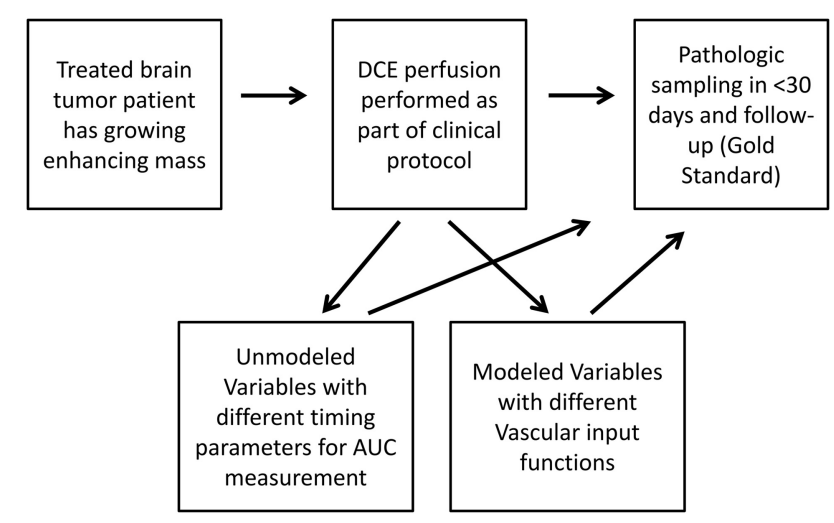

FIG 1. Overview diagram of the basic study design. This study tests the accuracy of DCE parameters in determining delayed radiation necrosis from recurrent glioma. Unmodeled parameters with varying times for the integration of DCE area under the curves and modeled parameters with varying locations/techniques for the vascular input function are tested. These are compared with the criterion standard of pathologic scoring and clinical follow-up.

centage difference in values and $\kappa$ statistics for the cutoff between grades 3 and 4 pathology reports.

\section{RESULTS}

\section{Patient Population}

Twenty-four patients met the inclusion criteria for the protocol, including biopsy within 30 days for suspicion of recurrent glioma with a progressively enhancing lesion on MR imaging. Of 200 patients evaluated with DCE under the protocol, 61 had biopsies. Twenty patients with biopsies were excluded because of a nonglioma primary tumor, no previous radiation therapy, or pathology sampling occurring $>30$ days after imaging. In addition, 17 patients were excluded due to a full-dose DCE examination (after an imaging protocol change). No patient was excluded due to the quality of the imaging.

There were 4 women and 20 men with an average age of $51 \pm$ 9 years. Patients had previous diagnoses of glioblastoma $(n=15)$, anaplastic astrocytoma $(n=7)$, anaplastic oligodendroglioma $(n=1)$, and oligodendroglioma $(n=1)$. At least 3 glioblastomas were secondary by dedifferentiation, with older pathologic results showing lower grade tumor. The average time from radiation therapy completion to imaging was $2.6 \pm 2.1$ years, though 2 patients had the protocol within 6 months of therapy completion. Thus, in most patients, the clinical question was recurrent glioma versus delayed radiation-induced necrosis (rather than pseudoprogression). All except 1 patient had previous treatment with temozolomide. Seven patients had previous treatment with bevacizumab, with a variety of other chemotherapies and/or vaccines used in 9 patients. An overview of the study design is given in Fig 1.

\section{Pathology Results and Follow-Up}

Median follow-up was 24 months, with 1 patient having $<3$ months' follow-up. Of the 15 tumor recurrences (pathology score of $1-3), 13$ patients (87\%) had died at the time of data analysis. Nine patients had delayed radiation-induced necrosis by pathology (pathology score of 4 or 5), of which only 2 patients (22\%) had died. There was a significant association between pathology 


\begin{tabular}{|c|c|c|c|c|c|c|c|}
\hline Measurement & Method & $\begin{array}{c}\text { Nonparametric } \\
\text { Coefficient }\end{array}$ & $\begin{array}{c}\text { Significance } \\
\text { ( } P \text { Value) }\end{array}$ & Post Hoc Cutoff & Sensitivity & Specificity & Accuracy \\
\hline Mean $K^{\text {trans }}$ & 1-Nordic & 0.606 & .002 & $\geq 0.05 \mathrm{~min}^{-1 \mathrm{~b}}$ & $80 \%$ & $78 \%$ & $79 \%$ \\
\hline Max $K^{\text {trans }}$ & 1-Nordic & 0.542 & .006 & $\geq 0.2 \mathrm{~min}^{-1}$ & $80 \%$ & $78 \%$ & $79 \%$ \\
\hline Mean $k_{e p}$ & 1-Nordic & 0.446 & .03 & $\geq 0.27 \mathrm{~min}^{-1}$ & $47 \%$ & $78 \%$ & $58 \%$ \\
\hline Mean $v_{p}$ & 1-Nordic & 0.555 & .005 & $>2^{c}$ & $71 \%$ & $89 \%$ & $79 \%$ \\
\hline $\operatorname{Max} v_{p}$ & 1-Nordic & 0.513 & .01 & $>9^{c}$ & $73 \%$ & $67 \%$ & $71 \%$ \\
\hline Mean $v_{e}$ & 1-Nordic & 0.566 & .004 & $>12^{c}$ & $80 \%$ & $78 \%$ & $79 \%$ \\
\hline Short AUC & 2-Simple & 0.410 & .047 & $>12 \%^{c}$ & $93 \%$ & $67 \%$ & $84 \%$ \\
\hline Intermediate AUC & 2-Simple & 0.478 & .018 & $>14 \%{ }^{c}$ & $93 \%$ & $67 \%$ & $84 \%$ \\
\hline Delayed short AUC & 2-Simple & 0.556 & .005 & $>20 \%{ }^{c, d}$ & $93 \%$ & $78 \%$ & $88 \%$ \\
\hline
\end{tabular}

Note:-Delayed short AUC indicates ratio of AUC from the lesion over the superior sagittal sinus vascular input integrated between the end of the initial vascular washout and early progressive leakage phases; max, maximum; $k_{e p}$, reflux rate constant; $v_{e}$, extravascular, extracellular volume fraction.

a The methods given are for model-independent "simple" calculations of the signal with time (method 2) versus the pharmacokinetic model calculations using nordiclCE (method 1). The pixel selection algorithm around the superior sagittal sinus was used for the latter. The nonparametric correlation to categoric ranking of pathology is given by a Spearman $\rho$ correlation. Post hoc arbitrary cutoff values are given for the most accurate performance for determining tumor, with tumor (pathology grading of 1-3) regarded as a positive case for sensitivity and specificity.

b Best performing modeled variable for correlation with pathology.

${ }^{c}$ A relative unit.

${ }^{\mathrm{d}}$ Best performing model independent variable.

score and overall survival (hazard ratio $=7.93$ for tumor recurrence, $P=.002$ ). Both evaluators of the pathology report were within a point for all cases. One specimen was changed from a pathology score of 3 to 2 after review of the specimen. There was no disagreement between scores of 3 and 4 .

\section{Image Acquisition and Analysis}

Method 1. To determine the best method for the nordicICE analysis (method 1), we tested 4 types of VIFs. The $K^{\text {trans }}$ values from region-of-interest pixel choosing from the MCA or SSS worked better than whole-brain coverage when compared with pathology. The fourth method for defining the VIF by using the complete region of interest in the SSS was immediately excluded because some of the maximum $K^{\text {trans }}$ values were reported as zero, which was not possible given the contrast enhancement of these areas indicating contrast escape from the vessel.

The Table demonstrates the best performing measurements for both methods 1 and 2. The best correlation to the pathologic grade by modeled values (method 1 ) was the mean extravascular extracellular volume fraction, the mean $v_{\mathrm{p}}$, and the mean $K^{\text {trans }}$ by VIF from pixel choosing in the SSS. Maximum $K^{\text {trans }}$, mean reflux rate constant, mean and maximum $v_{\mathrm{p}}$, and mean AUC also had significant correlations for values with VIF from the region of the SSS but not from the region of the MCA. An example of the variation based on the VIF is given in Fig 2. The mean $K^{\text {trans }}$ of $\geq 0.05$ minutes $^{-1}$ for residual tumor from the VIF near the SSS had 2 false-positives and 3 false-negatives (sensitivity $80 \%$, specificity 78\%, and accuracy 79\%). Although the model-independent values of scaled AUC and peak values did correlate to pathology, because they did not perform better than the best performing model-independent variable from method 2, they are not included in the Table.

Method 2. Of all the possible time iterations for the generation of normalized lesion AUC/VIF ratios (eg, wash-in to 90 seconds, washout to 60 seconds, and so forth), the integration from the end of the first-pass washout to 60 seconds after bolus arrival was the best model-independent measure correlated to the pathology grading (Spearman coefficient $=-0.56, P=.005$ ) and had the best accuracy of all measurements. The accuracy was 88\% (93\% sensitivity, $78 \%$ specificity) with 2 false-positives and 1 false-negative by using a cutoff value of $>20 \%$ for tumor. It was termed "delayed short AUC" because it fell between the initial vascular washout and progressive leakage phases. This value correlated with mean/maximum $K^{\text {trans }}$ (Spearman coefficient $=0.615 /$ $0.462, P=.001 / .021)$ and mean/maximum $v_{\mathrm{p}}$ (Spearman coefficient $=0.570 / 0.441, P=.004 / .031)$ by method 1 . The delayed short AUC also correlated to the mean unscaled area under the curve from method 1 (Spearman coefficient $=0.487, P=.016$ ); this outcome is expected because these are similar values without the use of model-dependent compartmental modeling.

The delayed short AUC outperformed the standard pharmacologic measurements of wash-in to 60 or 90 seconds (short and intermediate AUC, respectively). Graphic representations of delayed short AUC and intermediate AUC integrations used to derive these values are shown in Fig 3 in the case of tumor recurrence and Fig 4 for treatment-related necrosis. Note that the vascular input in the SSS on these figures happens to correspond to the axial image of the lesion location for ease of illustration, but this correspondence is often not the case. Also note, as explained in the legends, that the delayed short AUC by excluding the first pass of contrast is less sensitive to the large vessels, such as found in the VIF.

No additional arbitrary cutoff values for any of the parameters in method 1, with either the VIF from the SSS or MCA, performed as well as the delayed short AUC. The patients whose DCE values did not reflect pathology grading tended to have atypical treatment regimens such as prior vaccines or antivascular endothelial growth factor treatment (eg, bevacizumab). This finding is expected given the granulation tissue response, which can be created by locally delivered antitumor vaccines (false-positive) and the vascular normalizing effect of bevacizumab (false-negative). ${ }^{19}$

\section{Intra- and Interreader Variability}

For method 1, the second reader (J.L.) had a wide average variability of $49.8 \pm 286.1 \%$ from reader 1 (J.D.H.) for mean $K^{\text {trans }}\left(\kappa\right.$ value of 0 for cutoff of mean $K^{\text {trans }} \geq 0.05$ min- 

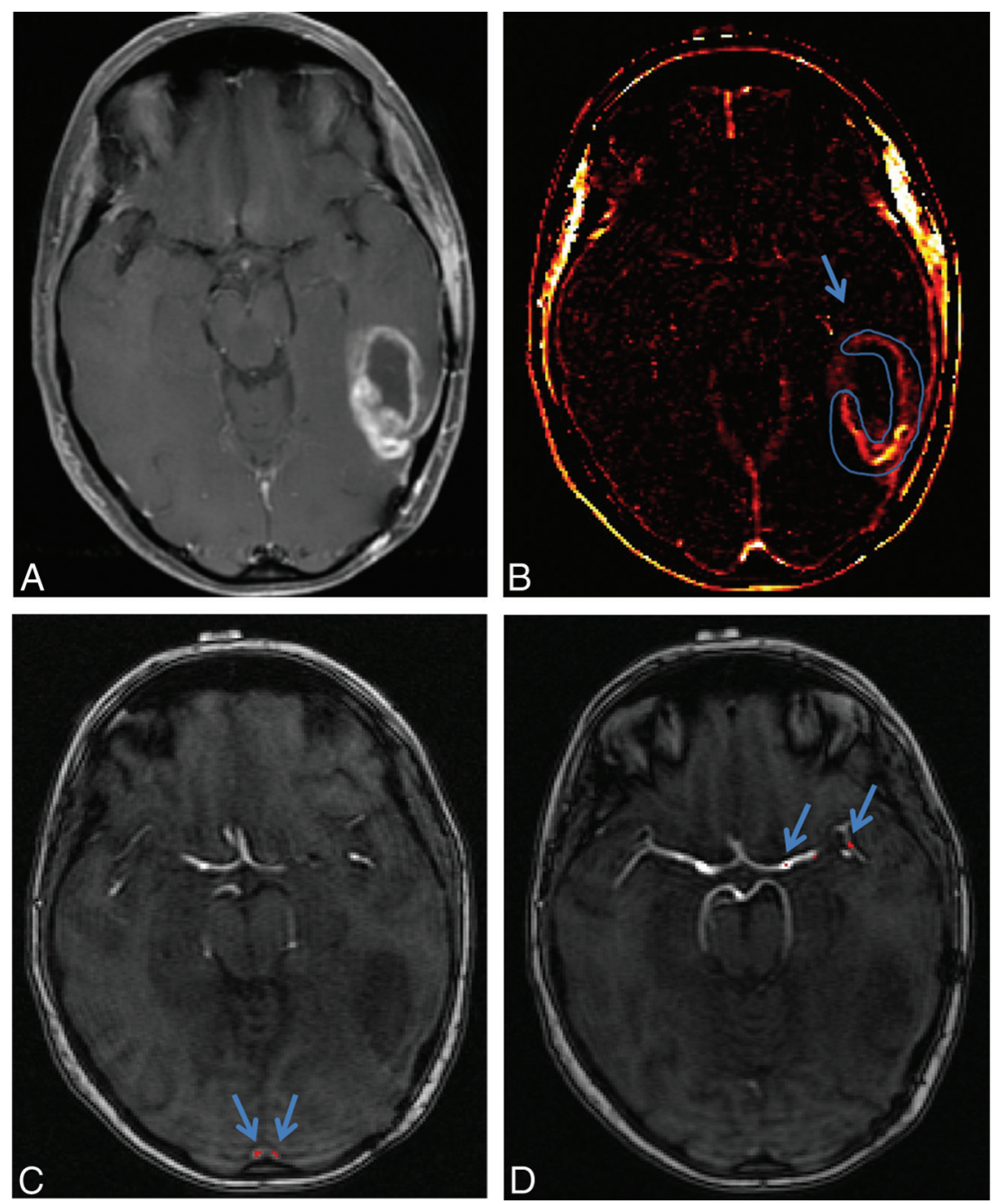

FIG 2. Example of a change in modeled values related to the location of the vascular input function. Note a left parietal ring-enhancing lesion on axial postcontrast $\Pi$ imaging $(A)$ and DCE $(B)$, which on subsequent pathology was recurrent glioblastoma with some superimposed treatment effects (pathology score of 2). A region of interest was drawn to cover the enhancing regions with sparing of the centrally necrotic portion. C, The pixels chosen by nordiclCE for a region of interest in the superior sagittal sinus. $D$, The pixels chosen for the $M 1$ and proximal $M 2$ branches of the ipsilateral middle cerebral artery. By changing from SSS to MCA, the mean/maximum $K^{\text {trans }}$ changed from 0.0165/0.169 to $0.283 / 3.003$ in relative units, a $20 \times$ difference. The mean/maximum plasma volume changed from $0.652 / 4.94$ to $5.49 / 34.87$, a nearly $10 \times$ difference.

utes $^{-11}$ ). For method 2, the second reader (C.I.) had $14.7 \pm$ $28.2 \%$ average variability for the short delayed AUC measurement compared with reader 1 (J.D.H). When the cutoff value of $20 \%$ was used, this yielded a $\kappa$ value of 0.6 , due to addition of 1 false-positive and 4 false-negatives. When the second reader repeated the measurement, there was $2.8 \pm 28.8 \%$ average intrareader variability.

\section{DISCUSSION}

We compared a simple model-independent signal-intensity change analysis versus a 2-compartment pharmacokinetic model analysis, choice of vascular input function, and choice of analysis output parameters to determine the optimal method for determining delayed radiation-induced necrosis from recurrent glioma. A novel measurement based on model-independent analysis performed in a manner equivalent to the corrected 2-compartment modeling analysis for our dataset. Because method 2 was processed on the same workstation as the rest of the protocol, there was a substantial decrease in analysis time. It took $\sim 10$ minutes for method 1 with additional time required to transfer images to a separate workstation compared with $\sim 5$ minutes for method 2 .

\section{Argument for Model-Independent Modeling}

There are several possible explanations for the delayed short AUC having worked well. First, not including the first pass of contrast agent results in relative resistance to the frequent issue of a large- or medium-sized vessel being within the same voxel or region of interest as the contrast-enhancing lesion (Figs 3 and 4). In some ways, this is like removing the signal usually gathered by gradient-echo dynamic susceptibility contrast perfusion on the first-pass of contrast for assessment of vascularity. Second, there is resistance to a greater value being generated primarily from the change in signal intensity occurring later due to slow progressive leakage, by having a relatively narrow range of integration (30-45 seconds). This is a characteristic of delayed radiation-induced necrosis (Fig 4). Third, by having the integration occur after the first-pass bolus and by using a venous rather than an arterial VIF, the tumor-related delay in mean transit time is less of an issue ${ }^{20}$ (see "Vascular Input Function Importance" section). Thus, the signal in the delayed short AUC primarily is derived from the contrast escape or permeability from the vessel derived during the first pass of contrast after the contrast in the vessel has washed out. To a lesser degree, delayed short AUC corresponds to the second pass of contrast within the vessels, which relates to the microvascular attenuation/ plasma volume. Thus, the observed signal change is a combination of pharmacokinetic model parameters $K^{\text {trans }}, v_{\mathrm{p}}$, and reflux rate constant, as demonstrated by the correlation with the modeled parameters.

Our technique tried to limit variations in contrast agent bolus, with most wash-in and washout periods averaging 3-4 time points or 15-20 seconds. The average delayed short AUC measures incorporated $\sim 9$ time points (45 seconds) of data. If the bolus was slow to washout on VIF, a minimum of 30 seconds of integration time was used. Delayed short AUC differs from the initial AUC by 60-90 seconds because it does not include the first pass of the contrast agent bolus. The latter have been reported in the pharmacologic literature as robust parameters without modeling error, ${ }^{14}$ but did not correlate as well in this study. 

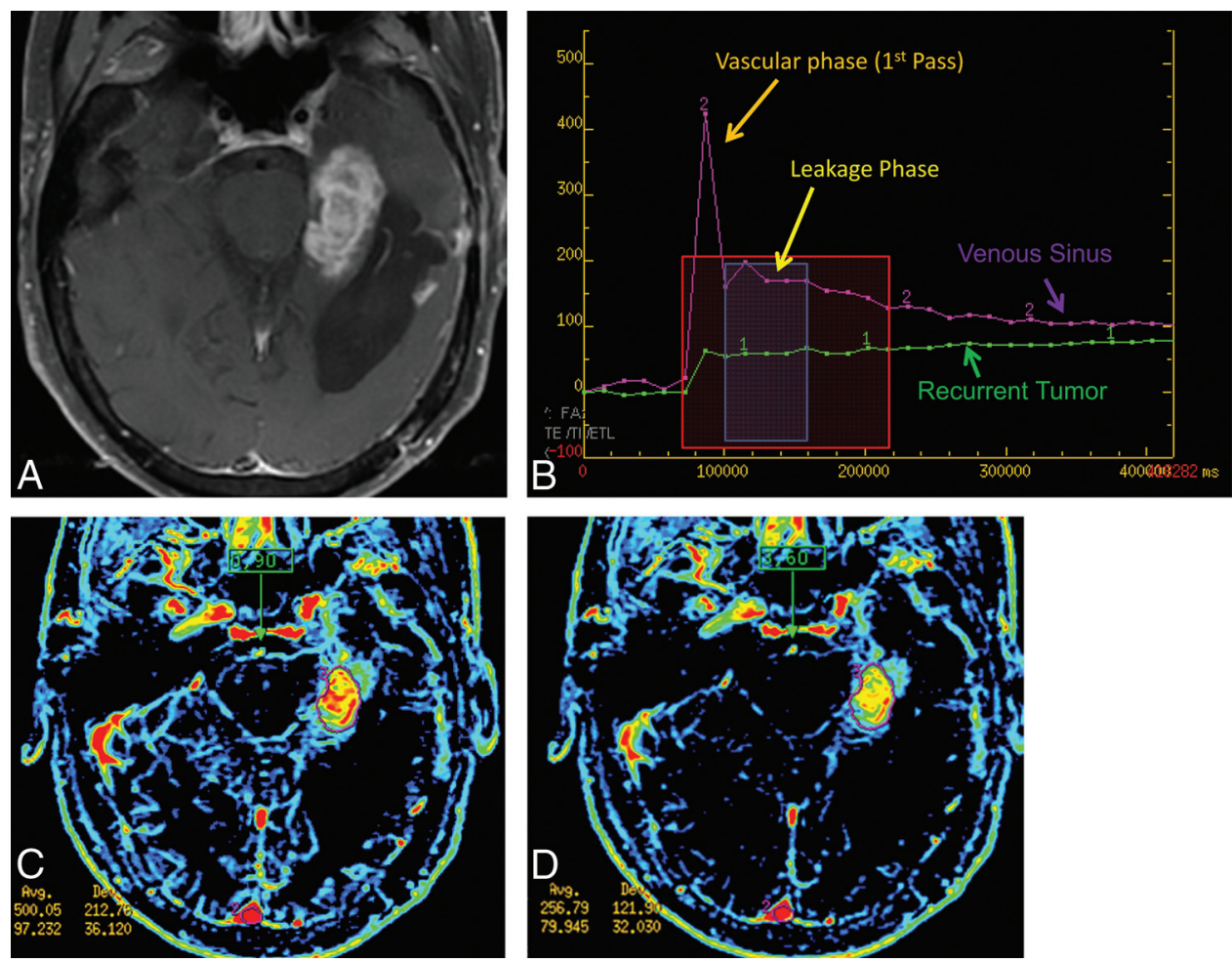

FIG 3. Example of recurrent glioblastoma by using 2 different AUC measurements. A, An enhancing lesion on TTWI involving the left parahippocampal region, which proved to be recurrent glioblastoma. B, A signal change over the time curve from the Advantage Workstation (GE Healthcare). The pink curve (voxel 2) is derived from the superior sagittal sinus (more inferior than normal positioning for illustration purposes) and demonstrates an initial vascular phase with the first pass of contrast washing in and then out. The green curve (voxel 1) demonstrates the signal change of a recurrent glioblastoma showing the initial rise of signal during the vascular phase followed by a slow rise during accumulated contrast escape or leakage of the contrast agent from the vessel. The red box demonstrates the time of integration for the "intermediate AUC" whose corresponding image is $C$, labeled "0.90." The blue box demonstrates the time of integration showing the values for the "delayed short AUC" over approximately 45 seconds, labeled "3.60." Notice in the corresponding image $(D)$ that the cortical vessels are less well-seen than in $C$ but the tumor remains (scaling is the same). Numerically, the vascular input decreases $49 \%$ (from an area under the curve of 500 to 257 relative units), while the tumor only decreases signal by $18 \%$ (97 to 80 ). Intermediate and delayed short AUC values from these data are $19 \%$ ( $97 / 500$ ) and $31 \%$ (80/257), respectively.

\section{Arguments against Simple Modeling}

One weakness of the delayed short AUC measurement is that it is heavily dependent on user input. The values for the SSS can be widely variable if care is not taken to do the following: 1) use a large enough region of interest to reduce undersampling, 2) keep the region of interest completely within the venous sinus, and 3) pick an area that minimizes inhomogeneity due to inflow from unopacified veins or sections toward the edges of the imaging volume. For this latter point, we used the vertical portion of the superior sagittal sinus in a region that did not have a feeding vein within the axial image. The delayed short AUC measurement also helped by using the vascular information in a more steady-state compared with the volatility and regional variability in signal change of the first pass of contrast. Additionally, the user must also define the lesion region of interest on the basis of the contrast enhancement, and this region of interest will invariably differ from observer to observer. ${ }^{21}$ These limitations are revealed in the interrater analysis, which shows wide variability and poorer performance by the second reader. Most surprising, the modeled data actually had greater variability, which may be partially attributed to the relative inexperience of the trainee. More sophisticated methods of volume rendering, segmentation, and histogram analysis have been reported, ${ }^{21}$ which may further improve the reproducibility of mean values from the ROIs.

Of the 5 recommendations for measurement of $K^{\text {trans }}$ or the initial area under the gadolinium concentration versus time curve made by a prior consensus group, ${ }^{14}$ this study did not use T1 mapping and estimates of contrast agent relaxivity in tumor vasculature and tissue for converting signal-intensity change to contrast agent concentration. Our group thought these steps would add further error/inefficiency and work against the goal of obtaining a simple, time-efficient data analysis methodology. However, a VIF and power injector were used to improve reproducibility in a group of patients with heterogeneous cardiac output. Also, scanning sufficiently began before contrast arrival to establish a baseline, at least 5 time points before contrast wash-in. These steps are necessary for model-independent measurements, which are more dependent on changes in the acquisition technique compared with complex modelbased analysis, ${ }^{14,21}$ which has been our experience. The magnet strength, injection parameters, cardiac output, and so forth can all have nonlinear changes in the dynamic $\mathrm{T} 1$ response that make it challenging to compare results from various institutions. ${ }^{14,21}$ Whatever the method chosen, consistency and pathologic validation are important for establishing it.

AJNR Am J Neuroradiol 36:686-93 Apr 2015 www.ajnr.org 

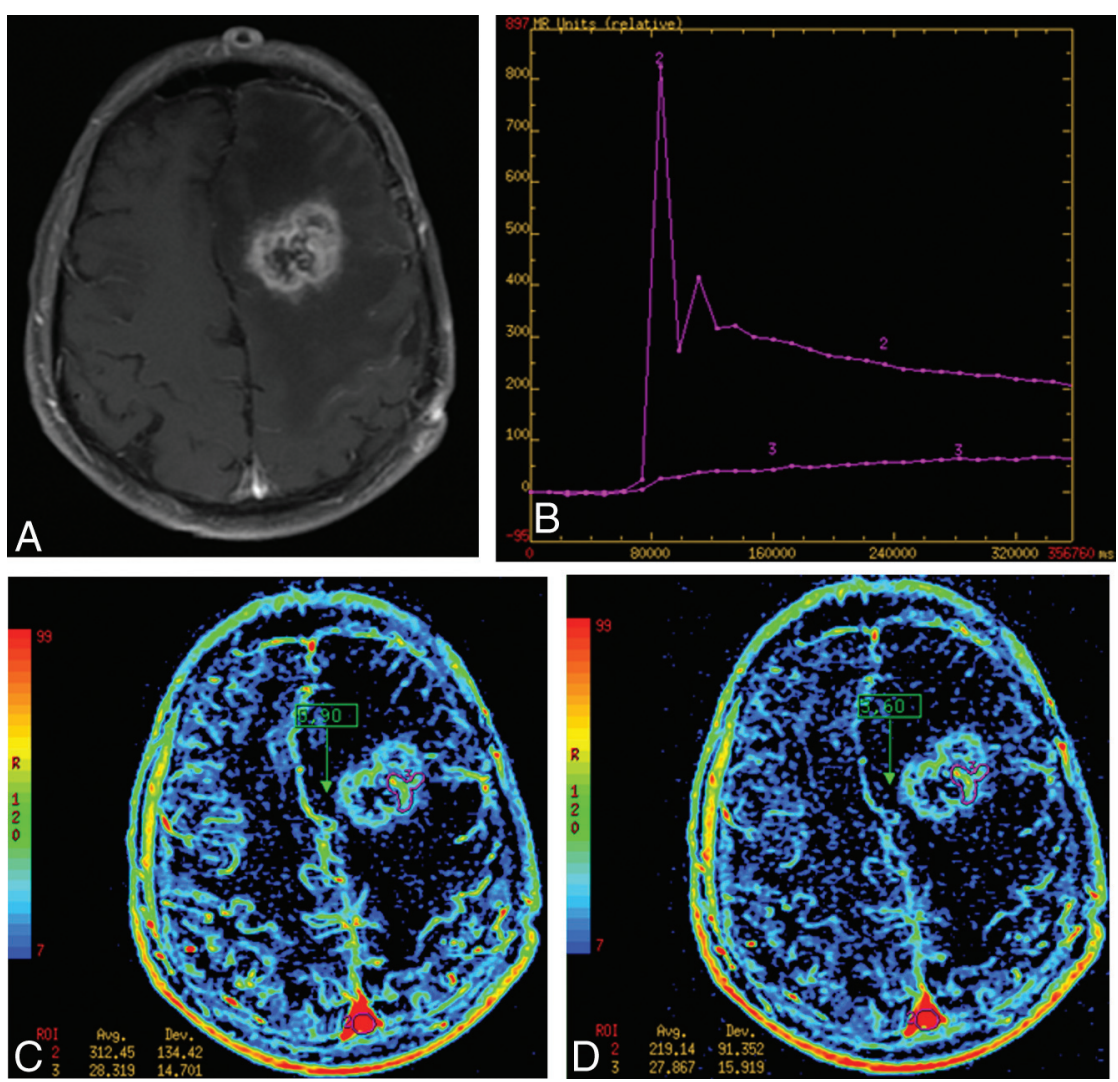

FIG 4. Example of treatment-related necrosis by using 2 different $A U C$ measurements. $A, A n$ enhancing lesion on T1WI of the left frontal lobe, which was proved to be treatment-related necrosis (pathology grade $=5$ ). $B$, A slow progressive increase in signal of the lesion compared with the vascular input and the lesion in B. C and D, the "intermediate AUC" and "delayed short AUC" integrations, respectively. Notice the significant drop in the superior sagittal sinus between these integrations (312 to 219 relative units) with little change in the lesion (it remains at 28). Intermediate and delayed short AUC values from these data are $9 \%(28 / 312)$ and $12 \%(28 / 219)$, respectively.

\section{Vascular Input Function Importance}

The method of VIF determination is important for determining clinically useful values. For this particular clinical question, the automated selection of pixels in a region near the superior sagittal sinus outperformed other locations for method 1 . We compared the timing of wash-in and washout of the first bolus pass from the MCA and SSS regions with the image mean value. In 11 of the 24 cases, there was a delay in the start of the wash-in and/or a delay in the peak of the VIF for the SSS compared with the image mean timing. Similarly, 5 of the cases had early wash-in or peak compared with the image mean timing with VIF around the MCA. On average, the peaks were also lower for the MCA compared with the SSS (596 \pm 461 versus $1457 \pm 1272$ in relative signal-intensity units, respectively). One possible reason that the VIF near the SSS worked better is that if there was to be a delay between the transit time of the lesion and the vein, there was still signal from contrast leakage and vascular recirculation in the enhancing lesion after the first pass. However, if the timing derived from the VIF was early, as with that derived from the MCA, this arrival may result in spuriously low-average results because the integration began before the first bolus pass reached the lesion and would have values near baseline T1. In other words, for the same increment of error in timing the VIF, there is probably less error in the measured values if it is late rather than early. The method of picking the VIF for the delayed short AUC was the least useful method when applied to method 1 , because it forced the $K^{\text {trans }}$ values to zero. This is likely because the SSS had such a large signal change that the contrast accumulation in the lesion was relatively insignificant in the model.

\section{Further Study Limitations}

A further limitation to any retrospective study is that the biopsy specimen location was not predetermined; therefore, the location could not be correlated directly back to a particular pixel or region-of-interest value. In addition, the pathology grading criteria were done retrospectively on the basis of reports and were confirmed in equivocal cases by pathology, rather than strict grading criteria, with actual percentage tumor estimations or automatic counts done prospectively. The total number of patients in this study was small. There was heterogeneity in the timing of imaging and the variety of treatments used.

\section{CONCLUSIONS}

Several methods of using DCE data were compared with the pathology for treatment-related effects versus recurrent high-grade gliomas; this comparison is relevant to clinical decision-making and survival. The results indicate that a measurement based on model-independent data analysis (delayed short AUC) performed at least as well as more complex model-based methods, with an accuracy of up to $88 \%$. Because this measurement is technique- and observer-dependent, care must be taken to ensure reliability. Additionally, it is shown that vascular input function derived from the superior sagittal sinus is superior to that from the middle cerebral artery.

\section{ACKNOWLEDGMENTS}

We thank Dawid Schellingerhout, MB, ChB, and Kelly Duggan, The University of Texas M.D. Anderson Cancer Center; and Sandeep N. Gupta, PhD, GE Global Research Center, for their help in making this manuscript possible.

Disclosures: Claro Ison—UNRELATED: Employment: Cardinal MRI Center

\section{REFERENCES}

1. Ostrom QT, Gittleman H, Liao P, et al. CBTRUS statistical report: primary brain and central nervous system tumors diagnosed in the United States in 2007-2011. Neuro Oncol 2014;16(suppl 4):iv1-iv63

2. Ballman KV, Buckner JC, Brown PD, et al. The relationship between six-month progression-free survival and 12-month overall survival end points for phase II trials in patients with glioblastoma multiforme. Neuro Oncol 2007;9:29-38 
3. Yang I, Aghi MK. New advances that enable identification of glioblastoma recurrence. Nat Rev Clin Oncol 2009;6:648-57

4. Kumar AJ, Leeds NE, Fuller GN, et al. Malignant gliomas: MR imaging spectrum of radiation therapy- and chemotherapy-induced necrosis of the brain after treatment. Radiology 2000;217:377-84

5. Kohshi K, Imada H, Nomoto S, et al. Successful treatment of radiation-induced brain necrosis by hyperbaric oxygen therapy. J Neurol Sci 2003;209:115-17

6. Levin VA, Bidaut L, Hou P, et al. Randomized double-blind placebo-controlled trial of bevacizumab therapy for radiation necrosis of the central nervous system. Int J Radiat Oncol Biol Phys 2011;79:1487-95

7. Gonzalez J, Kumar AJ, Conrad CA, et al. Effect of bevacizumab on radiation necrosis of the brain. Int J Radiat Oncol Biol Phys 2007;67:323-36

8. Weller M, Cloughesy T, Perry JR, et al. Standards of care for treatment of recurrent glioblastoma: are we there yet? Neuro Oncol 2013;15:4-27

9. Bisdas S, Naegele T, Ritz R, et al. Distinguishing recurrent highgrade gliomas from radiation injury: a pilot study using dynamic contrast-enhanced MR imaging. Acad Radiol 2011;18:575-83

10. Narang J, Jain R, Arbab AS, et al. Differentiating treatment-induced necrosis from recurrent/progressive brain tumor using nonmodelbased semiquantitative indices derived from dynamic contrast-enhanced T1-weighted MR perfusion. Neuro Oncol 2011;13:1037-46

11. Barajas RF Jr, Chang JS, Segal MR, et al. Differentiation of recurrent glioblastoma multiforme from radiation necrosis after external beam radiation therapy with dynamic susceptibility-weighted contrast-enhanced perfusion MR imaging. Radiology 2009;253:486-96

12. Hu LS, Baxter LC, Smith KA, et al. Relative cerebral blood volume values to differentiate high-grade glioma recurrence from posttreatment radiation effect: direct correlation between imageguided tissue histopathology and localized dynamic suscepti- bility-weighted contrast-enhanced perfusion MR imaging measurements. AJNR Am J Neuroradiol 2009;30:552-58

13. Zeng QS, Li CF, Liu H, et al. Distinction between recurrent glioma and radiation injury using magnetic resonance spectroscopy in combination with diffusion-weighted imaging. Int J Radiat Oncol Biol Phys 2007;68:151-58

14. Leach MO, Brindle KM, Evelhoch JL, et al. The assessment of antiangiogenic and antivascular therapies in early-stage clinical trials using magnetic resonance imaging: issues and recommendations. Br J Cancer 2005;92:1599-610

15. Tofts PS, Kermode AG. Measurement of the blood-brain barrier permeability and leakage space using dynamic MR imaging. 1. Fundamental concepts. Magn Reson Med 1991;17:357-67

16. Murase K, Yamazaki Y, Miyazaki S. Deconvolution analysis of dynamic contrast-enhanced data based on singular value decomposition optimized by generalized cross validation. Magn Reson Med Sci 2004;3:165-75

17. Mouridsen K, Christensen S, Gyldensted L, et al. Automatic selection of arterial input function using cluster analysis. Magn Reson Med 2006;55:524-31

18. Sourbron S, Ingrisch M, Siefert A, et al. Quantification of cerebral blood flow, cerebral blood volume, and blood-brain-barrier leakage with DCE-MRI. Magn Reson Med 2009;62:205-17

19. Pope WB, Young JR, Ellingson BM. Advances in MRI assessment of gliomas and response to anti-VEGF therapy. Curr Neurol Neurosci Rep 2011;11:336-44

20. Bjornerud A, Sorensen AG, Mouridsen K, et al. T1- and T2*-dominant extravasation correction in DSC-MRI: part I-theoretical considerations and implications for assessment of tumor hemodynamic properties. J Cereb Blood Flow Metab 2011;31:2041-53

21. Jackson A, O'Connor JP, Parker GJ, et al. Imaging tumor vascular heterogeneity and angiogenesis using dynamic contrast-enhanced magnetic resonance imaging. Clin Cancer Res 2007;13:3449-59 